\title{
EL NEODARWINISMO Y LAS CIENCIAS DE LA FORMA: EL PORQUÉ DE UNA ALIANZA INICIAL IMPOSIBLE
}

\author{
Miquel DE RENZI \\ Departament de Geologia e Institut Cavanilles de Biodiversitat i Biologia \\ Evolutiva. Universitat de València. Campus de Burjassot. Burjassot, 46100 \\ Valencia
}

De Renzi, M. 1999. El Neodarwinismo y las ciencias de la forma: el porqué de una alianza inicial imposible. [Neodarwinism and the sciences of form: The reason of an impossible initial alliance]. Revista Española de Paleontología, n* extr. Homenaje al Prof. J. Truyols, 115-120. ISSN 0213-6937.

\begin{abstract}
The Modern Synthesis has been very reluctant to introduce development and morphology among its main arguments. An important divorce with sciences of form (morphology and embryology) arose at its beginning, when selection and adaptation coloured pervasively the theoretical framework of the modern evolutionary theory. Although there were divergences between embryologists and geneticists, many of the former were prepared to understand the role of genes in development and then, to supply ideas in the growing evolutionary theory. On the other hand, the shape of the organisms was only interpreted in terms of selection-adaptation as the only laws ruling it. Any internalist position was qualified as "idealistic morphology" and then, antievolutionary. A more comprehensive point of view would be necessary in order to built a more complete synthesis. Possible unconscious ideological causes are pointed out to understand these missing viewpoints: reminiscences of natural theology and the opposite view to the marxist determinism: history is contingent.
\end{abstract}

Keywords: History of Science, Neodarwinism, Morphology, Embryology.

\section{RESUMEN}

La Síntesis Moderna ha estado muy poco dispuesta a introducir el desarrollo y la morfología entre sus principales argumentos. Ya desde el comienzo, surgió un importante divorcio con las ciencias de la forma, puesto que selección y adaptación penetraron todo el edificio teórico de la teoría evolutiva moderna. Aunque desde muy pronto hubo diferencias entre embriólogos y genéticos, muchos de los primeros estaban dispuestos a comprender el papel de los genes en el desarrollo y, en consecuencia, a proporcionar ideas en la naciente teoría evolutiva. Por otra parte, la teoría sintética interpretó la forma de los organismos, casi exclusivamente, en términos de selección-adaptación. Cualquier postura internalista fue calificada de "morfología idealista" y, por ello mismo, como antievolutiva. Habría sido necesario un punto de vista más amplio a fin de construir una síntesis más completa. Se han considerado algunas posibles causas ideológicas no asumidas conscientemente a fin de explicar tales ausencias: reminiscencias de teología natural y una concepción opuesta al determinismo marxista: la historia es contingente.

Palabras clave: Historia de la Ciencia, Neodarwinismo, Morfología, Embriología.

Deseo aprovechar la ocasión que ofrece este homenaje al Profesor D. Jaime Truyols para destacar una faceta muy importante de su labor científica. Esta se refiere a que, tanto él como su maestro, el Profesor Crusafont, percibieron algo que ya se iba perfilando en el seno de la comunidad paleontológica internacional, pero que todavía iba a tardar años en ser tomado en consideración y dar sus frutos: la necesidad de volver a contemplar los fósiles como testimonios de una vida pretérita y, por tanto, retomar su significación paleobiológica.

En los años 40 surgió la Síntesis Moderna de la evolución, y al Profesor Truyols le cupo el honor de participar en el equipo de trabajo de los "Cursillos Internacionales de Paleontología" de los años 1952, 1954 y 1956 que, con la evolución orgánica como leit-motiv, organizó el Profesor Crusafont. Fue el reunir hombres como los paleontólogos Jean Piveteau, el director de uno de los primeros tratados modernos de paleontología sistemática, o Björn Kurtén, un gran especialista en mamíferos fósiles y uno de los introductores de los conceptos modernos de paleoecología, un mérito indiscutible de Crusafont y de ese grupo de entusiastas que se asoció con él, en un momento en 
que en España no soplaban vientos muy favorables para hablar sobre evolución.

Aunque la Síntesis representó uno de los primeros intentos de gran alcance para recuperar el enfoque biológico en paleontología, hasta la década de los sesenta, que yo recuerde, no se editó un manual de paleontología con dichas perspectivas: el de Beerbower (1960). Este hecho es significativo, toda vez que los libros de texto son los propagadores de los paradigmas científicos. Sin embargo, el Profesor Truyols ya había avanzado en esa línea de un modo valiente, bien fuera asociado al Profesor Crusafont en un trabajo de 1956 (Crusafont y Truyols, 1956), que planteó los aspectos biométricos de la evolución en los carnívoros fisípedos, cuyo interés continúa vigente a juzgar por las citas que todavía he podido ver del mismo en algunas revistas de gran prestigio. Bien fuera en una magnífica síntesis, completamente al día para su época, acerca de los usos de los procedimientos biométricos y estadísticos en el estudio de la evolución orgánica, como la que figura en el primer libro en castellano que se publicó sobre evolución en la postguerra (Crusafont et al., eds., 1966), el cual fue un punto de referencia inicial y esencial para todos los que nos estábamos formando en ese momento.

Estos aspectos pioneros del Profesor Truyols ahora pueden quedar ocultos por una importante labor posterior, pero creo que merecen un reconocimiento explícito, ya que contribuyeron, desde nuestro país, a poner de manifiesto la necesidad de esos nuevos supuestos que tanto requería la paleontología.

\section{INTRODUCCIÓN}

La paleontología es una ciencia con una doble vertiente: trata con evidencias de antiguos seres vivos y de su organización, registradas en los estratos sedimentarios, con lo cual entra en el marco de las disciplinas de la biología. Pero también se integra dentro de aquel contexto que Charles Lyell definió como geología, dentro de la cual, el cambio orgánico ocupa un papel tan importante como el físico para comprender la historia de la Tierra y los procesos que actuaron en ella. Uno de los aspectos esenciales a tratar se refiere a la evolución orgánica, uno de los campos en que la paleontología tiene más que decir y aportar al campo de la biología.

Los fósiles, una vez descodificados los efectos de los procesos tafonómicos, exhiben unos caracteres primarios eminentemente morfológicos. Los paleontólogos, desde los tiempos de Cuvier, en los albores de la biología moderna, nos hemos preocupado de la significación de esa forma y, como mínimo, por su origen individual. No podemos dejar de recordar que Étienne Geoffroy SaintHilaire, Karl Ernst von Baer, etc. fueron embriólogos (estudiosos del origen individual de los organismos) y paleontólogos (Rudwick, 1972). También hay que decir que una de las preocupaciones de Charles Darwin era la de explicar los cambios morfológicos por los cuales unas especies se modificaban para dar origen a otras. En estos comienzos ya hallamos una vieja disputa que cabría ilustrar aparte: las estructuras orgánicas suelen estar implicadas en funciones, y puede sobrevenir la tentación de intentar explicarlas en términos exclusivamente funcionales y negar que existan estructuras sin función. Esta fue la posición de Georges Cuvier. Sin embargo, también se puede plantear que primero hay que dar razón de las mismas y luego pensar en las funciones que pueden ejercer. Tal punto de vista lo mantuvo Étienne Geoffroy Saint-Hilaire (Radl, 1988a).

El propósito de este trabajo es mostrar que si bien se ha afirmado que nada consistente puede ser dicho en biología si no es a la luz de la teoría de la evolución, ello puede tener serias contrapartidas si dicha teoría no tiene unos fundamentos coherentes. Un ejemplo muy primario de ello podemos hallarlo en la famosa formulación de Haeckel acerca de que la ontogenia recapitula mecánicamente la filogenia. Haeckel veía en la filogenia (resultado de la evolución) un mecanismo suficiente para explicar los procesos embriológicos. Con ello detuvo la investigación experimental en embriología hasta finales del siglo pasado, en que Wilhelm Roux prescindió por completo de tales planteamientos (Gould, 1977a).

La Síntesis Evolutiva Moderna puso, hacia los años 50 , el acento en la adaptación como motor evolutivo (Gould, 1980). De este modo, las formas no estarían sometidas a otras limitaciones que las impuestas por las necesidades funcionales de los organismos. Los cambios de éstos se habrían de dar en el sentido adaptativo y, si no, la selección los eliminaría. Ello quiere decir que cualquier cosa es posible previamente. Sólo en años recientes se ha intentado poner coto a una concepción tan ambientalista y se ha tratado de valorar el peso de los llamados factores internos (Gould, 1977b), que parece ser mucho mayor de lo que se sospechaba. Tal planteamiento ya comenzó con las ideas del paleontólogo alemán Adolph Seilacher (cf. su versión actualizada en Seilacher, 1991), contenidas en su concepto de morfología construccional, y con las aportaciones más recientes sobre las relaciones entre desarrollo y evolución, como las de Alberch' (1982, 1991), Edelman (1988) u otros. Sin embargo, el rechazo de las ciencias de la forma, como posible luz para la comprensión de los mecanismos evolutivos, se inicia ya al principio de la Síntesis y en ello parece volver a alentar la vieja disputa entre Cuvier y Geoffroy Saint-Hilaire. Algunos biólogos sinteticistas, así como también algunos filósofos e historiadores de la ciencia defensores del neodarwinismo quisieron explicar históricamente tal rechazo en el marco de un muy conocido libro (Mayr y Provine, 1980), aunque de un modo bastante tendencioso. Aquí serán analizadas sus razones y se intentará poner de manifiesto las causas subyacentes de tal modo de ver las cosas.

${ }^{1}$ En el momento de introducir las modificaciones sugeridas por los revisores, se cumplen cuatro meses de la muerte de Pere Alberch. De este modo, este trabajo se convierte en un recordatorio de algunos de los puntos de vista que él defendió. Aquí se han citado dos trabajos suyos; los que deseen conocer más a fondo sus ideas tienen un listado amplio en De Renzi (1998). 


\section{CÓMO SE INTERPRETABA LA SITUACIÓN DE LAS CIENCIAS DE LA FORMA EN EL MOMENTO EN QUE SURGIÓ LA SÍNTESIS}

Varios autores han hecho una valoración de las dos principales ciencias de la forma - la morfología y la embriología (que hoy también conocemos como biología del desarrollo) - en relación con la teoría sintética. Son, entre otros, Coleman (1980), Ghiselin (1980) y Hamburger (1980). Se puede decir que tal valoración se asienta en un estudio histórico realizado por los tres autores, pero que parte de una sola premisa: la selección es el único modo de explicación válido en evolución orgánica. Si es así, disciplinas tales como la morfología y la embriología (e igualmente la paleontología) únicamente tienen como misión ilustrar tal principio (Ghiselin, op. cit.). En ese sentido, Hamburger (op. cit.) afirma muy claramente que la ausencia de la biología del desarrollo en la Síntesis Evolutiva Moderna no debe ser objeto de crítica a los fundadores de ésta, puesto que it has always been a legitimate and sound research strategy to relegate to a "black box", at least temporarily, wide areas that although pertinent would distract from the main thrust (p. 99).

Por otra parte, la morfología sería una ciencia que habría renunciado a planteamientos autónomos. Según constata Coleman (1980), su única contribución a la concepción evolucionista, en la primera mitad de este siglo, estuvo en los aspectos comparativos, y su mayor interés se centró en el tema de las homologías. Así, Hyman afirmaba en el año 1922 (fide Coleman, op. cit., p. 174): The whole aim of comparative anatomy is to discover what structures are homologous, pero ésta es también la postura de Romer (y otros), que además añade posibles explicaciones en términos de adaptación y genética, pero sin profundizar en ello ( fide Coleman, op. cit.).

Los autores aquí citados, pues, no destacan como contribuciones de las ciencias de la forma a la Síntesis, más que aquéllas que apunten a una clara explicación en términos adaptativos o funcionales; es decir, aquéllas que se basen en el mecanismo de la selección natural. Tal es el caso del ruso Sewertzoff, inicialmente seguidor de Haeckel, pero buen investigador de los vertebrados inferiores, tanto desde un punto de vista embriológico como filogenético (fide Ghiselin, 1980). Aunque este autor, en principio, justificara la ley biogenética, las numerosas excepciones que hallaba a la misma le inclinaron hacia interpretaciones funcionales y ecológicas, que implicarían más bien un mecanismo selectivo y no uno "endógeno" (entrecomillado de Ghiselin, op. cit.). Parece ser que Sewertzoff tuvo una ascendencia considerable entre los evolucionistas rusos y, por ello, también en Dobzhansky, ruso de origen.

Si no existen mecanismos endógenos para explicar evolutivamente la forma o su origen individual (desarrollo), huelga decir que todo aquello que pretenda explicar la morfología desde ese punto de vista es remitido a la peyorativa (por estática) etiqueta de "morfología idealista"2. No otra cosa quiere decir Ghiselin (1980) cuando afirma que el orden subyacente a la vida quiso ser buscado en un contexto no biológico, que sería el de la física y el de la química, y creo que aquí hay una implícita referencia a D'Arcy Wentworth Thompson. El razonamiento de Ghiselin va en el sentido de que la física y la química tienen una estructura legaliforme bien fundada y que las leyes de la naturaleza son de origen divino, un punto de vista que se mantuvo durante el s. XIX y que podría estar en relación con aquellas fuerzas creativas del paleontólogo alemán Georg-Heinrich Bronn, cuyo carácter no sería vital sino físico, y que serían responsables del origen de las especies en el tiempo obedeciendo a unas leyes muy concretas (Rudwick, 1972). De aquí que Ghiselin (op. cit.) afirme que ese hablar de leyes conduciría a una visión estática de la naturaleza que haría innecesario ser evolucionista.

Es cierto que el azar es algo característico de la evolución y del pensamiento evolucionista; en esto lleva razón Ghiselin (1980). Si consideramos que las leyes no permiten contingencias de ningún tipo, estamos en una visión puramente estática ya que todo está determinado de antemano. Pero las cadenas causales, completamente determinadas cada una de ellas por distintas leyes, son independientes, y ello genera esa contingencia propia de la evolución. Luego, hablar de evolución y de ley no es contradictorio. Mediante leyes y su articulado formal tratamos de construir las teorías científicas, que constituyen el máximo grado del conocimiento científico (cf. Bunge, 1976; capítulos 6, 7 y 8) y de esto Ghiselin (op. cit.) parece ser consciente al final (pp. 187-188). Es cierto que la biología ha de tener explicaciones legítimas para dar razón de las estructuras de los organismos; según Ghiselin (op. cit., p. 191), éstas habría que buscarlas en la economía y no en la física; tal cosa, pues, impondría un lenguaje propio, común a ambas disciplinas (ver también Ghiselin, op. cit., p.182). Ahora bien, ello indica algo en común entre los sistemas biológicos y económicos, que es que escapan a las explicaciones meramente mecanicistas que el paradigma newtoniano ha impuesto a la mentalidad occidental. Pero no es menos cierto que en cualquier sistema existen diversos niveles de integración y que las leyes de los niveles más nuevos no pueden de ninguna manera entrar en contradicción con las de aquellos más antiguos (Bunge, 1976; pp. 355-357). Y, como ya muy bien dijo Thompson (1942; sus ideas básicas ya aparecieron en la primera edición de On growth and form; su autor afirma en el prefacio de la segunda edición que sólo efectuó una revisión de la primera, pero no en sus aspectos esenciales; sin embargo, dicha segunda edición fue considerablemente ampliada con respecto a la primera; Witowski, 1998): In short, the form of an object is a "diagram of forces" [...] . In an organism, great or small, it is not merely the nature of the motions of the living substance which we must interpret

${ }^{2}$ La morfología idealista - término en contraposición a la anatomía evolutiva de Haeckel - tiene por finalidad la investigación de las formas de los organismos en términos de figuras geométricas ideales, en cuya definición estuvieran contenidas todas sus propiedades y, de aquí, la idea platónica de la cual derivan. Ahora bien, esto impulsó un programa de investigación que culminó con hallazgos tales como la espiral logarítmica para las conchas de los moluscos o la filotaxis para los vegetales (cf. Radl, 1988b; pp. 20-48). 
in terms of force (according to kinetics), but also the conformation of the organism itself, whose permanence or equilibrium is explained by the interaction or balance of forces, as described in statics (p. 16).

Comentar a fondo la obra de Thompson (op. cit.) se saldría mucho de este marco, pero la necesidad de tenerla en consideración ya ha sido puesta de manifiesto por algunos neodarwinistas. Así, Frazzetta (1975), en un esfuerzo por conciliar el aspecto puramente físico de la materia viviente con el modelo vigente de la evolución en aquel entonces, se refería a la obra de Thompson como one of the truly great masterworks of biology. En una etapa todavía adaptacionista y neodarwinista de su pensamiento, Gould (1971) hace también una justa defensa de muchos aspectos de la manera de pensar de Thompson.

\section{¿QUÉ PREDISPOSICIÓN HABÍA REALMENTE?}

Hamburger (1980) nos da a entender que la postura de los biólogos del desarrollo frente a la evolución no fue tan negativa, aunque en principio pareciera haber distancias insalvables, sobre todo en lo referente al papel de la genética, lo cual, irónicamente, contradice su restante argumentación. Ésta se refiere, de entrada, a un divorcio entre los biólogos del desarrollo y los genéticos durante la primera mitad del siglo $\mathrm{XX}$, puesto que la idea general era que el material hereditario permanecía invariable en el núcleo, mientras que durante el desarrollo las células presentaban notables diferenciaciones en sus citoplasmas. Por tal motivo, la idea de muchos embriólogos era que los aspectos causales del desarrollo radicaban no en el genoma uniforme sino en el citoplasma, cuya enorme diferenciación ya se hacía patente en el zigoto. Desde luego, tal creencia era completamente razonable para esa época. La única excepción al respecto fue la de Morgan (fide Hamburger,1980), que sí creía que genética y embriología habían de ir a la par, porque sus historias estaban entrelazadas de una manera muy estrecha. Si la genética era una de las claves de la Síntesis Moderna, tal divorcio negaba la posibilidad de que la ciencia del desarrollo se integrara en la misma.

Además, Hamburger (1980; p. 104) destaca también que Driesch, en 1894, ya había visto algo de lo que ahora es tan familiar para aquellos que estamos interesados en las relaciones entre desarrollo y evolución: que núcleo y citoplasma interactuarían; que las sustancias determinantes se originarían en el citoplasma bajo control nuclear (por tanto, no serían liberadas por el núcleo), y que el estado del citoplasma sería el causante de nuevas acciones nucleares que conducirían a subsiguientes diferenciaciones en aquél. Ahora bien, según Hamburger, estas hipótesis no ganaron adeptos en la época en que fueron publicadas. Sin embargo, y aquí radica la contradicción de esta historia whig (en el sentido de estar explicada de tal modo que conduzca inevitablemente al estado actual de cosas, que es el que ha tenido éxito), el mismo autor señala que una de las figuras fundamentales de la embriología de los años 20, Spemann, afirmaba en 1924: the activation of the genome does not occur by autonomous segregation of the hereditary factors but under far-reaching interaction between the parts, hence epigenetically (fide Hamburger, 1980; p. 103). Esto es, había una estrecha relación entre la localización de los factores hereditarios en el embrión y su activación. Por otra parte, la misma fuente relata que a la hora del té, al final de los años 20 y principio de los 30, había discusión sobre el tema de la evolución en el laboratorio de Spemann.

Posteriormente, Huxley trató de relacionar embriología y genética en sus estudios sobre crecimiento diferencial (Huxley, 1972; la edición que manejo es una reimpresión no abreviada de la original de 1932, con una introducción escrita especialmente por el autor en 1971). En esta obra, muy anterior al establecimiento definitivo de la Síntesis, se toca el tema del fundamento genético de las alometrías (aquí todavía denominadas heterogonías): los rate-genes de Goldschmidt, así como su relación con la evolución en sentido seleccionista (capítulo VII, pp. 204-244). Pero Julian Huxley también fue uno de los fundadores de la Síntesis. Hamburger (1980) también menciona su libro de 1942: Evolution: the modern synthesis (no lo he podido consultar directamente), en donde Huxley afirma: Any originality which this book may possess lies partly in furthering Fisher's ideas and partly by stressing the fact that a study of genes during development is as essential for an understanding of evolution as are the study of mutation and selection (fide Hamburger, op. cit.; p. 99). Está claro que la posibilidad de relacionar desarrollo con genética y desarrollo con evolución estaba en la mente de muchas de las figuras relevantes de la biología que trabajaron entre los años 20 y 30. Únicamente en el lado ruso, Schmalhausen fue capaz de tratar la cuestión de cómo la selección actuaría sobre los genes controlantes de la variabilidad de los procesos de desarrollo, pero a causa del aislamiento entre Este y Oeste durante la Segunda Guerra Mundial, su influencia llegó demasiado tarde (hacia 1949; fide Hamburger, 1980).

Finalmente, Hamburger (1980) comenta el libro de Waddington (1957) como el esquema del "capítulo ausente" de la Síntesis en lo referente al desarrollo. Sin embargo, el libro en cuestión pone el acento en cosas tales como estados de equilibrio engendrados por la acción química de los genes, los únicos estados finales alcanzables a través del desarrollo. Ahora bien, parece que lo que pensaba Waddington (op. cit.) de muchas de las cosas que afirmaban en ese momento los constructores de la Síntesis no era muy halagüeño para estos últimos; así: As it stands, the first part of it, 'natural selection', is a tautology, and the second part, 'random variation', an equivocation (p. 64). Este pensamiento es muy semejante al formulado por Thompson (1942; p. 8).

\section{EPÍLOGO}

Lo dicho hasta ahora muestra una sordera, por parte de los promotores de la Teoría Sintética, en lo referente a tratar con ciertos temas. Una primera cuestión a la cual no se ha querido prestar atención, es la causación intrínseca de las formas de los organismos, como si no obedecieran a más 
leyes que a las impuestas por los requerimientos ambientales o por su papel funcional. Esto es indicador de un punto de vista netamente seleccionista o adaptacionista. Cualquier actitud que apunte a la búsqueda de causas intrínsecas es tildada de morfología idealista o de estar atada a los modos de pensar del siglo XIX. Incluso se critica la morfología funcional (The resulting "functional morphology", even when comparative, tended to ask physiological questions, and cultivated physical-science thought habits to the detriment of biological ones; Ghiselin, 1980; p.191). Esto me hace pensar en una disputa entre químicos en que unos acusaran a otros de hablar de fuerzas eléctricas en vez de usar unos conceptos (y un lenguaje) exclusivamente químicos ${ }^{3}$. Hay como un retorno a la vieja disputa entre Cuvier y Geoffroy Saint-Hilaire. En la Síntesis parece que se alza triunfante el antievolucionista (por escepticismo positivista) Cuvier.

No obstante lo dicho, fue esa "morfología idealista" que había predicado Thompson en su gran On growth and form, la que contribuyó a la renovación en paleontología evolutiva. Así, los estudios de alometría realizados por Simpson (1984; he podido disponer de esta reciente reimpresión del original de 1944, con una nueva introducción del propio Simpson), combinando datos ontogénicos del caballo con datos de los fósiles de adultos de la misma familia, que asimismo cita nuestro homenajeado (Truyols, 1966), son derivaciones de las ideas de Thompson, que Huxley (1972) siempre reconoció, tal como indica la dedicatoria de su libro. Sólo la esclerotización tardía de la Síntesis produjo la pérdida de tan importantes puntos de vista, con su acento sobre selección dirigida hacia la adaptación (Gould, 1980).

Ahora bien, la causación de las formas tiene dos facetas: el proceso que las produce y su estado final, que ha de obedecer a una serie de requisitos. El proceso que las produce no es otro que el desarrollo embrionario; éste ya fue visto, de un modo clarividente por Thompson (1942) y Waddington (1957), como un proceso en el que tendrían un papel muy importante los mecanismos fisicoquímicos. Quizá la clave del rechazo de estos aspectos está, inicialmente, en que en todo este tipo de explicaciones, la selección natural no se erige en fuerza principal y tiene que conformarse con aquello que está permitido como compatible con las leyes de la física y de la química, que representaría el componente interno. Una de las frases finales de Ghiselin (1980) es altamente reveladora: This point was driven home in discussion by Hamburger, who remarked how hard he found it to conceive of natural selection acting on the inside of an organism (p. 191). Es decir, según estos autores, si hubiera de existir algún factor

\footnotetext{
${ }^{3}$ No obstante esto, hay algo más. Ghiselin (1980) (ver más arriba) predecía la unión de la biología y la economía para convertirse en una misma rama del conocimiento (p. 182). Yo veo en esto un intento de privar a la biología de su carácter autónomo, algo que Thompson (1942) nunca hizo. La afirmación de Ghiselin (op. cit.) se contradice, además, con algo que dice en otro lugar, como una acusación: que el orden subyacente a la vida fue buscado en un contexto no biológico, como es el de la física y el de la química.
}

interno, éste sería la selección natural, lo cual es imposible.

Así vemos cómo algunos relevantes textos anteriores sobre evolución, escritos desde una óptica neodarwinista, soslayan el tema del desarrollo o dicen acerca de él vaguedades conocidas de todo el mundo en una sola página; por ejemplo, Grant (1971). Textos más recientes, como Ayala (1982), omiten todo tratamiento que no sea genético. Únicamente el mencionado texto de Frazzetta (1975) se asoma de un modo decidido a esta temática, aunque tratando de justificar siempre la naturaleza gradual del cambio orgánico.

Por tanto, el planteamiento de una explicación de carácter único en evolución ha hecho que se arroje al bebé con el agua sucia por el desagüe. Han sido desechados hechos pertinentes con tal de no desviar la atención de los investigadores con respecto a un punto central de la teoría. Otra vez, una postura monista ha desbaratado la posibilidad de construir una teoría rica en consecuencias. Como un irónico retorno a Haeckel.

Desearía, finalmente, hacer un par de consideraciones sobre el origen, posiblemente inconsciente, de ese monismo. En primer lugar, la actitud antifísica de aquellos teóricos que se oponen a una concepción interna de la forma. Es talmente como si la física hubiera de supeditarse a algún tipo de finalidad en los seres vivos. Podría ser indicativo de un retorno a posiciones inspiradas por la teología natural. No deja de tener relevancia que el darwinismo y la posterior Síntesis Moderna de la Evolución nacieran en países del área anglosajona, y que la evolución darwinista sea, inicialmente, una concepción producida en un mundo netamente protestante. Si la teología natural afirmaba que la naturaleza no jugaba (cf. Rudwick, 1972, acerca de la discusión sobre la naturaleza de los fósiles en Inglaterra), la física tiene poco que decir de las estructuras orgánicas, expresamente diseñadas para funciones específicas. La selección deviene creadora y reemplaza la acción directa divina.

En segundo lugar: la historia es contingente, y el acercamiento a una física de las formas eliminaría tal contingencia (Ghiselin, 1980; p. 191), aunque ello hoy día se muestra como una verdadera exageración. La Síntesis, no lo olvidemos, nace no sólo en un área protestante. También lo hace en un campo que habría de ser campeón de los principios capitalistas. La esclerosis de la Síntesis se inicia en la década de los 50 (cf. Gould, 1980; pp. 165-168). Puede ser coincidencia, pero es la etapa del comienzo de la guerra fría. Si la historia es contingente, queda negada cualquier vinculación con el determinismo marxista. La historia de la vida también habría de estar libre de todo determinismo. Sería un mero juego de individualidades, nunca por encima del organismo.

\section{AGRADECIMIENTOS}

Deseo expresar las gracias a los dos revisores de este trabajo: el Dr. Luis Carlos Sánchez de Posada (Dpto. de Geología, Universidad de Oviedo) y el Dr. Sixto Fernández López (Dpto. de Paleontología, Universidad Complutense de 
Madrid), cuyos comentarios me han ayudado a mejorar el trabajo.

\section{BIBLIOGRAFÍA}

Alberch, P. 1982. Developmental constraints in evolutionary processes. In: Evolution and development (Ed. J.T. Bonner). Dahlem Konferenzen 1982. Springer-Verlag, Berlin, Heidelberg, New York, 313-332.

Alberch, P. 1991. Del gen al fenotipo: sistemas dinámicos y evolución morfológica. Revista Española de Paleontología, no extr., 13-19.

Ayala, F.J. 1982. Population and evolutionary genetics: A primer. The Benjamin/Cummings Publishing Company, Inc. Menlo Park, California; Reading, Massachusetts; London; Amsterdam; Don Mills, Ontario; Sydney, 268 pp.

Beerbower, J.R. 1960. Search for the past. An introduction to paleontology. ( $1^{\mathrm{a}}$ ed.). Prentice Hall, Inc. Englewood Cliffs, New Jersey, 562 pp.

Bunge, M. 1976. La investigación científica. Su estrategia y su filosofía. ( $5^{\mathrm{a}} \mathrm{ed}$.). Editorial Ariel. Barcelona, Caracas, México, $955 \mathrm{pp}$.

Coleman, W. 1980. Morphology in the evolutionary synthesis. In: The evolutionary synthesis. Perspectives on the unification of biology (Ed. E. Mayr and W.B. Provine). Harvard University Press. Cambridge, Massachusetts, and London, England, 174-180.

Crusafont, M., Meléndez, B. and Aguirre, E. (Eds.) 1966. La evolución. Biblioteca de Autores Cristianos, n. 258. Madrid, $1014 \mathrm{pp}$.

Crusafont, M. and Truyols, J. 1956. A biometrical study of the evolution of fissiped carnivores. Evolution, 10, 314-332.

De Renzi, M. 1998. Recordando a Pere Alberch, biólogo evolutivo. Noticias Paleontológicas, 31, 53-58.

Edelman, G.M. 1988. Topobiology. An introduction to molecular embryology. Basic Books, Inc., Publishers. New York, 240 pp.

Frazzetta, T.H. 1975. Complex adaptations in evolving populations. Sinauer Associates, Inc. Publishers. Sunderland, Massachusetts, $267 \mathrm{pp}$.

Ghiselin, M.T. 1980. The failure of morphology to assimilate darwinism. In: The evolutionary synthesis. Perspectives on the unification of biology (Ed. E. Mayr \& W.B. Provine). Harvard University Press. Cambridge, Massachusetts, and London, England, 180-193.

Gould, S.J. 1971. D'Arcy Thompson and the science of form. In: Topics in the philosophy of biology (Ed. M. Grene \& E. Mendelsohn). D. Reidel Publishing Company. Dordrecht-Holland y Boston (U.S.A.), 66-97.
Gould, S.J. 1977a. Ontogeny and Phylogeny. The Belknap Press of Harvard University Press. Cambridge, Massachusetts and London, England, 501 pp.

Gould, S.J. 1977b. Eternal metaphors of palaeontology. In: Patterns of evolution, as illustrated by the fossil record (Ed. A. Hallam). Elsevier Scientific Publishing Company. Amsterdam, Oxford, New York, 1-26.

Gould, S.J. 1980. G.G. Simpson, paleontology, and the modern synthesis. In: The evolutionary synthesis. Perspectives on the unification of biology (Ed. E. Mayr \& W.B. Provine). Harvard University Press. Cambridge, Massachusetts, and London, England, 153-172.

Grant, V. 1971. The origin of adaptations. Columbia University Press. New York and London, 606 pp.

Hamburger, V. 1980. Embryology and the modern synthesis in evolutionary theory. In: The evolutionary synthesis. Perspectives on the unification of biology (Ed. E. Mayr and W.B. Provine). Harvard University Press. Cambridge, Massachusetts, and London, England, 97-112.

Huxley, J. 1972. Problems of relative growth. Dover Publications, Inc. New York, 312 pp.

Mayr, E. and Provine, W.B., Eds. 1980. The evolutionary synthesis. Perspectives on the unification of biology. Harvard University Press. Cambridge, Massachusetts, and London, England, 487 pp.

Radl, E.M. 1988a. Historia de las teorías biológicas. 1. Hasta el siglo XIX. Alianza Universidad, n. 553. Madrid, 334 pp.

Radl, E.M. 1988b. Historia de las teorías biológicas. 2. Desde Lamarck y Cuvier. Alianza Universidad, n. 554. Madrid, $425 \mathrm{pp}$.

Rudwick, M.J. S. 1972. The Meaning of Fossils. Episodes in the History of Palaeontology. History of Science Library. Macdonald, London and American Elsevier Inc., New York, 287 pp.

Seilacher, A. 1991. Self-organizing morphogenetic mechanisms as processors of evolution. Revista Española de Paleontología, $\mathbf{n}^{\mathbf{0}}$ extr., 5-11.

Simpson, G.G. 1984. Tempo and mode in evolution (Reimpresión). Columbia University Press. New York, $237 \mathrm{pp}$.

Thompson, D'A.W. 1942. On growth and form. ( $2^{\mathrm{a}}$ ed.). Cambridge University Press, England, 1116 pp.

Truyols, J. 1966. Parámetros numéricos en la evolución orgánica. In: La evolución (Eds. M. Crusafont, B. Meléndez y E. Aguirre). Biblioteca de Autores Cristianos, n. 258. Madrid, 385-415.

Waddington, C.H. 1957. The strategy of the genes. A discussion of some aspects of theoretical biology. George Allen \& Unwin, LTD. London, $261 \mathrm{pp}$.

Witowski, N. 1998. D'Arcy Thompson un excéntrico de la biología. Mundo Científico, 188, 44-47. 